\title{
Managing your wine fermentation to reduce the risk of biogenic amine formation
}

\section{Anita Yolandi Smit, Lynn Engelbrecht and Maret du Toit*}

Institute for Wine Biotechnology, Department of Viticulture and Oenology, Stellenbosch University, Stellenbosch, South Africa

Edited by:

Giovanna Suzzi, Università degli Studi

di Teramo, Italy

\section{Reviewed by:}

Giovanna Suzzi, Università degli Studi di Teramo, Italy

Javier Carballo, University of Vigo,

Spain

*Correspondence:

Maret du Toit, Institute for Wine

Biotechnology, Stellenbosch

University, Private Bag X1, Matieland

7602 Stellenbosch, South Africa.

e-mail:mdt@sun.ac.za
Biogenic amines are nitrogenous organic compounds produced in wine from amino acid precursors mainly by microbial decarboxylation. The concentration of biogenic amines that can potentially be produced is dependent on the amount of amino acid precursors in the medium, the presence of decarboxylase positive microorganisms and conditions that enable microbial or biochemical activity such as the addition of nutrients to support the inoculated starter cultures for alcoholic and malolactic fermentation (MLF). MLF can be conducted using co-inoculation or an inoculation after the completion of alcoholic fermentation that may also affect the level of biogenic amines in wine. This study focused on the impact of the addition of complex commercial yeast and bacterial nutrients and the use of different MLF inoculation scenarios on the production of biogenic amines in wine. Results showed that the addition of complex nutrients to real grape must could potentially increase histamine concentrations in wine. The same experiment in synthetic grape must showed a similar trend for putrescine and cadaverine. The effect of different MLF inoculation scenarios was examined in two cultivars, Pinotage and Shiraz. Conflicting results was obtained. In the Shiraz, co-inoculation resulted in lower biogenic amine concentrations after MLF compared to before MLF, while the concentration was higher in the Pinotage. However, the production of biogenic amines was affected more by the presence of decarboxylase positive lactic acid bacteria than by the addition of complex nutrients or the inoculation scenario.

Keywords: biogenic amines, nutrients, co-inoculation, malolactic fermentation, lactic acid bacteria, wine

\section{INTRODUCTION}

Biogenic amines are basic nitrogenous compounds produced in wine mainly through the decarboxylation of amino acids by yeasts or lactic acid bacteria (LAB). The concentration of biogenic amines that can potentially be produced in wine largely depends on the abundance of amino acid precursors in the medium, the presence of decarboxylase positive microorganisms and wine parameters such as $\mathrm{pH}$, alcohol, and sulfur dioxide that will impact the growth of microbes (Smit et al., 2008; Moreno-Arribas et al., 2010).

Vintage, grape variety, geographical region, and vinification methods such as grape skin maceration are some of the variables that can lead to an increase of precursor amino acids and subsequently the biogenic amine content in wine. Aging of wine on yeast lees involves autolyzing yeast cells that release vitamins and nitrogenous compounds into the wine. The latter may include amino acids that are the precursors of biogenic amines (Smit and Du Toit, 2011).

The yeasts and $\mathrm{LAB}$ responsible for wine fermentations have certain basic nitrogen nutrient requirements. Saccharomyces species can utilize the ammonium ion $(\mathrm{NH} 4+)$ and free alpha amino acids as nitrogen sources. Moreover, yeasts can synthesize all required nitrogen compounds, including amino acids, from ammonium. However, if amino acids are present yeasts will use it very efficiently after ammonium has been depleted. Yeast strains display different preferences for the uptake of different amino acids, and can also secrete certain amino acids into the wine (Bely et al., 1990). In general, yeasts require at least a minimum of $140-150 \mathrm{mg} / \mathrm{N} / \mathrm{L}$ to prevent stuck fermentations, but $200 \mathrm{mg} / \mathrm{L}$ is recommended to avoid the formation of off-flavors (RibérauGayon et al., 2006). Because ammonium alone does not meet all the nutritional requirements of yeast, many wine yeast manufacturers recommend the use of complex yeast nutrients that include a nitrogen supplement (González-Marco et al., 2006; HernándezOrte et al., 2006). Bach et al. (2011) have shown that the addition of yeast nitrogen compounds leads to an increase in the total level of biogenic amines in wine.

Lactic acid bacteria require and are able to use only complex organic nitrogen sources, such as amino acids. They can also utilize peptides or proteins as nitrogen sources by the breakdown to amino acids by proteolytic enzyme activity (Leitão et al., 2000). It has been shown that the highest risks for the production of histamine and tyramine is during malolactic fermentation (MLF; Vidal-Carou et al., 1990a,b; Soufleros et al., 1998; van der Merwe, 2007). Generally complex malolactic nutrients include inactivated yeast cells rich in alpha amino acids as well as casein, vitamins, minerals, polysaccharides, and cellulose. As with commercial yeast preparations, commercial malolactic nutrients are recommended for use with fermentation starter cultures (selected Oenococcus oeni strains) unable to produce biogenic amines according to the manufacturers (Lerm et al., 2010). However, in practice winemakers 
might add nutrients to sluggish spontaneous MLF or allow spontaneous MLF to proceed after complex yeast nutrients had been added during alcoholic fermentation (AF). The question arises whether residual precursor amino acids from complex nutrients could be present in the wine for the natural LAB flora, which could include decarboxylase positive strains, to use.

In this study, the influence of complex commercial yeast and bacterial nutrients on biogenic amine production by yeast and natural LAB (partially supplemented by decarboxylase positive Lactobacillus species) in the wine or added to a synthetic medium are evaluated. The second aim was to assess the impact of different MLF inoculation scenarios on the production of biogenic amines.

\section{MATERIALS AND METHODS GRAPE MUST FERMENTATIONS WITH COMPLEX NUTRIENTS}

Cabernet Sauvignon grapes from the Paarl region and Shiraz grapes from the Stellenbosch region, South Africa, were used in this study. After grapes were destemmed and crushed, the skins and free-run juice were separated and homogenized. Equal amounts of free-run juice (per volume) and grape skins (per weight) were allocated to each treatment in $10 \mathrm{~L}$ plastic buckets. Sulfur dioxide $\left(\mathrm{SO}_{2}\right)$ was added to the must of all treatments at $20 \mathrm{mg} / \mathrm{L}$. AF was performed by Saccharomyces cerevisiae strain NT202 (Anchor Yeast, South Africa) in all treatments; rehydrated and inoculated at $30 \mathrm{~g} / \mathrm{hL}$ according to the instructions of the manufacturer. Sugar density was measured daily in all treatments with a Brix hydrometer to monitor the progression of AF, which was conducted at room temperature. The temperature in the cellar was not controlled, but grape must temperature was recorded daily and fluctuated between 19 and $22^{\circ} \mathrm{C}$. Grape skins were punched down daily throughout $\mathrm{AF}$ and pressed with a hydraulic basket press to 1 bar at the completion of AF. Wines were transferred to $4.5 \mathrm{~L}$ glass bottles to complete MLF at $20^{\circ} \mathrm{C}$. MLF was performed spontaneously by strains native to the grapes used in this study, supplemented with a culture containing confirmed decarboxylase positive strains isolated from spontaneous MLF in South African wine (Downing, 2003; Smit et al., submitted). This decarboxylase positive culture, comprised of equal cell concentrations of Lactobacillus hilgardii B74, L. hilgardii M59, and Lactobacillus brevis M58 (Smit et al., submitted), was inoculated into all treatments at approximately $10^{6} \mathrm{CFU} / \mathrm{mL}$ after the completion of $\mathrm{AF}$ and prior to the start of spontaneous MLF.

Representative samples for the analysis of biogenic amines and microbial enumeration were drawn in sterile sample vials before AF (grape must), after AF, and after MLF. The presence and growth of LAB in the wine was monitored by plate counts on selective agar media (Smit and Du Toit, 2011). MLF was monitored using Fourier transform mid infrared spectroscopy (FT-MIR; WineScan FT120, FOSS Analytical, Denmark) to determine malic acid and lactic acid concentrations in the wine. All treatments were repeated in duplicate for each of the two cultivars. Treatments consisted of the addition of commercial preparations of complex yeast or bacterial nutrients to the fermenting must or wine. Yeast nutrients were added after the exponential growth phase of yeast; $1-3$ days after inoculation with yeast for AF, as recommended by the manufacturers. Bacterial (malolactic) nutrients were added after AF, before the start of MLF. All complex nutrients were added to the fermenting must or wine at the maximum dosage recommended by the manufacturers. Treatment 1 contained no added nutrients, treatments 2 , 3 , and 4 were supplemented with complex yeast nutrients (nutrients $A, B$, and C), and treatments 5 and 6 were supplemented with complex bacterial nutrients (nutrients D and E).

The following descriptions of the compositions of the nutrients used in this study were provided by the manufacturers. Nutrient A contains inactivated yeast, diammonium phosphate (DAP), and ammonium sulfate. Nutrient B contains inactivated yeast, DAP, and one specific vitamin. Nutrient $C$ contains inactivated yeast, DAP, vitamins, minerals, unsaturated fatty acids, and sterols. Nutrient D contains inactivated yeast and cellulose. Nutrient $\mathrm{E}$ contains inactivated yeast, cellulose, and casein. It is not clear in all cases from the manufacturers' descriptions whether vitamins, minerals (trace elements), polysaccharides, sterols, and fatty acids were added or derived from the inactivated yeast. Some manufacturers claim to use specially selected inactivated yeast.

\section{SYNTHETIC MEDIUM FERMENTATIONS WITH COMPLEX NUTRIENTS}

Synthetic grape must (MS300) described by Bely et al. (1990) was used to perform a similar experiment to confirm results under controlled conditions. The following modifications were made to the synthetic grape must: $120 \mathrm{mg} / \mathrm{L}$ ammonium chloride was included as the only nitrogen source (no amino acids were added as per the original protocol), $0.005 \%$ of pyridoxal $5^{\prime}$-phosphate (SigmaAldrich, Germany) was added to induce decarboxylase activity. All chemicals used for the preparation of the synthetic grape must were purchased from Saarchem (Merck, South Africa) except for malic acid, glucose, and ammonium chloride (Sigma-Aldrich, Germany).

The treatments in the synthetic grape must consisted of four control treatments without the addition of any complex nutrients, (1) in the absence of yeast and $\mathrm{LAB},(2)$ in the presence of yeast and LAB, (3) in the absence of yeast but presence of LAB, and (4) in the absence of LAB but presence of yeast. Treatments 5-10 consisted of different combinations of three complex yeast and two bacterial nutrients; (5) nutrients $\mathrm{A}$ and $\mathrm{D},(6)$ nutrients $\mathrm{A}$ and $\mathrm{E}$, (7) nutrients $B$ and D, (8) nutrients $B$ and E, (9) nutrients $C$ and $\mathrm{D}$, and (10) nutrients $\mathrm{C}$ and $\mathrm{E}$. Treatments 11 (nutrient $\mathrm{D}$ ) and 12 (nutrient E) contained only bacterial nutrients.

Saccharomyces cerevisiae strain NT202 was rehydrated and inoculated at $30 \mathrm{~g} / \mathrm{hL}$ for AF. Complex yeast nutrients were added to the fermenting synthetic must $48 \mathrm{~h}$ after the start of AF. Fermentations were performed under static conditions in $100 \mathrm{~mL}$ Erlenmeyer flasks fitted with airlocks, at $30^{\circ} \mathrm{C}$. After 14 days $\mathrm{AF}$ was considered complete when $\mathrm{CO}_{2}$ gas formation seized, and yeast cells were removed from the treatments by centrifugation at $8000 \mathrm{rpm}$ for $5 \mathrm{~min}$ to prevent further fermentation or autolysis and release of nutrients from the yeast cells. Complex bacterial nutrients were added to the synthetic wine and $1 \%$ of precultured decarboxylase positive LAB culture was inoculated into the medium. LAB growth (with limited MLF) proceeded for a period of 14 days at $30^{\circ} \mathrm{C}$.

Prior to the start of this experiment, the five nutrients tested, dissolved in synthetic must, were analyzed for biogenic amines. Samples were drawn from the fermentation medium after AF and at the end of growth of LAB for biogenic amine analysis. 
CO-INOCULATION VERSUS SEOUENTIAL MALOLACTIC FERMENTATION Malolactic fermentation was carried out in the cultivars Pinotage and Shiraz to examine the influence of different MLF inoculation times on the biogenic amine content of wine. The co-inoculation treatments were inoculated for MLF $24 \mathrm{~h}$ after the addition of the yeast for $\mathrm{AF}$, whereas the sequential treatments were inoculated for MLF by the conventional practice, after the completion of AF. All treatments were performed in triplicate, using $9.5 \mathrm{~kg}$ of grapes for the co-inoculation fermentations and $4.5 \mathrm{~L}$ of wine for the sequential fermentations. After destemming and crushing of the grapes, sulfur dioxide was added at a concentration of 20 and $30 \mathrm{mg} / \mathrm{L}$ to the co-inoculation and sequential treatments, respectively. The S. cerevisiae yeast strain WE372 $(30 \mathrm{~g} / \mathrm{hL}$; Anchor Yeast, South Africa) was used for AF. The juice was supplemented with diammonium phosphate $(250 \mathrm{mg} / \mathrm{L})$ on day one of AF. No additional nutrients were added to any treatment. AF was performed at $25^{\circ} \mathrm{C}$. As soon as AF was completed (residual sugar $<5 \mathrm{~g} / \mathrm{L}$ ) the wines were moved to a $20^{\circ} \mathrm{C}$ incubation room to complete MLF. After the completion of MLF (which ranged between 18 and 34 days) sulfur dioxide was added to a final concentration of between 60 and $80 \mathrm{mg} / \mathrm{L}$, after which the wines were bottled. The MLF treatments for the Pinotage consisted of a spontaneous control, which was not inoculated for MLF, two treatments inoculated with L. hilgardii strains (L. hilgardii 1 and L. hilgardii 2 ) and one treatment inoculated with a Lactobacillus plantarum strain. These Lactobacillus cultures were isolated from South African wines and were investigated for their ability to perform MLF (unpublished data). For the Shiraz, a treatment inoculated with a commercial O. oeni starter culture was included for comparison to the Lactobacillus cultures. Samples were taken from all fermentations before inoculation for MLF and again at the completion of MLF for analyses of the biogenic amines histamine, tyramine, putrescine, and cadaverine.

Statistically significant differences between the amine concentration before and after MLF for a specific inoculation time were evaluated by one-way analysis of variance with Fisher's LSD test at a $95 \%$ significance level. The analysis was performed using Statistica (Stat Soft, Inc., USA, version 10).

\section{BIOGENIC AMINE ANALYSES}

The biogenic amines histamine, tyramine, putrescine, and cadaverine were quantified by two different methods in this study. For the small scale wine fermentations with complex nutrients, biogenic amines were analyzed by high performance liquid chromatography (HPLC) using the method described by Alberto et al. (2002) with modifications (Smit and Du Toit, 2011). Amines in the synthetic media fermentations with complex nutrients, and in coinoculation versus sequential MLFs were determined using liquid chromatography mass spectrometry (LC-MS/MS) as described by Smit et al. (submitted).

\section{RESULTS \\ GRAPE MUST FERMENTATIONS WITH COMPLEX NUTRIENTS}

On average, AF was complete in 9 days in Cabernet Sauvignon and 8 days in Shiraz. Figures $\mathbf{1}$ and $\mathbf{2}$ show the results of malic acid degradation and growth of LAB during the course of MLF in the two cultivars. In Cabernet Sauvignon (Figure 1), MLF initially proceeded faster in some treatments that received added complex nutrients. When comparing the three treatments that received additional complex yeast nutrients (treatments 2, 4, and 4); not only was the rate of AF enhanced (data not shown), but treatments 2 and 3 completed spontaneous MLF at a faster rate (44-51 days) than the control, treatment 1 (66 days). MLF in treatments 5 and 6 also proceeded faster than in the control treatment (59 days), suggesting that malolactic nutrients can act to increase the initial rate of spontaneous MLF. Treatment 4 (yeast nutrient C) did not enhance MLF rate in Cabernet Sauvignon. For Shiraz treatments the rate of MLF was similar in all treatments (Figure 2), and took approximately 40 days to be completed. There were no significant differences between LAB numbers measured in relation to the nutrient treatments.

Four of the biogenic amines commonly found in wine were analyzed (histamine, tyramine, putrescine, and cadaverine). No tyramine was detected in any of the treatments for both cultivars. Although biogenic amines were detected in the grape must (putrescine), and wines after AF and MLF (putrescine and cadaverine), levels were similar for all treatments and no effect of nutrient addition could be observed (results not shown). The only biogenic amine that showed a difference between treatments was histamine. Figure 3 shows the concentrations of histamine detected in samples of the grape must, at the end of AF and at the end MLF in Figure 3A Cabernet Sauvignon and Figure 3B Shiraz treatments.

\section{SYNTHETIC MEDIUM FERMENTATIONS WITH COMPLEX NUTRIENTS}

Figure 4 shows the concentrations of each biogenic amine detected in samples of the synthetic must, at the end of AF and at the end of the 14-day growth period of LAB in the synthetic wine.

Biogenic amines were absent or detected only at very low levels in the synthetic grape must with or without dissolved complex nutrients.

When no LAB were present (treatments 1 and 4), no significant production of any biogenic amines was measured in this study.

It seems as if though the Lactobacillus species produced much higher levels of histamine and tyramine when their growth was not preceded by the growth and fermentation of yeast (treatment 3). Also, the absence of any complex nutrients seemed to stimulate histamine and tyramine formation by Lactobacillus species (treatment 3). Similar concentrations (for both histamine and tyramine) were reached for all treatments in the presence of both yeast and bacteria (treatments 5-12) regardless of whether complex nutrients were absent from the medium (treatment 2) or present in any combination or alone.

More putrescine was produced in treatments that were supplemented with complex nutrients than in control treatments 2 and 3 containing Lactobacillus species but no added nutrients. No significant amounts of putrescine were produced in the absence of LAB (treatments 1 and 4). Therefore, all of the tested nutrient combinations had an influence on putrescine production, with bacterial nutrients added alone (treatments 11 and 12) leading to a slightly higher production when compared to any combination of yeast and bacterial nutrients. It appears that the yeast NT202 produced low levels of cadaverine during AF (treatments 2 and 4), more so in the presence of complex yeast nutrients (treatments 5-10). After the introduction of Lactobacillus species an increase of cadaverine 

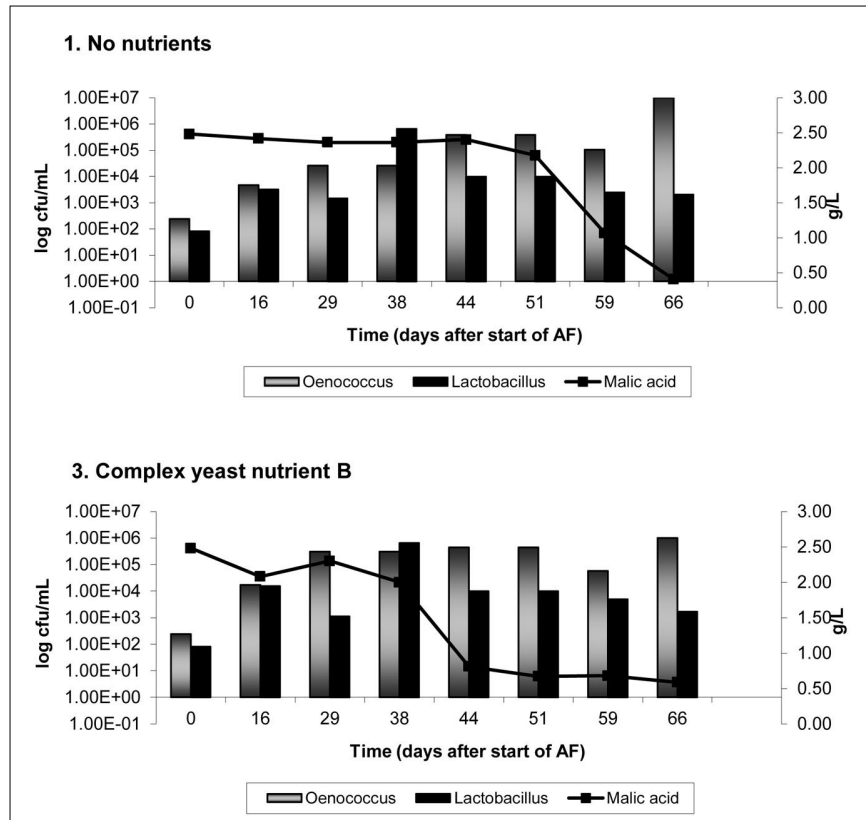

5. Complex MLF nutrient D

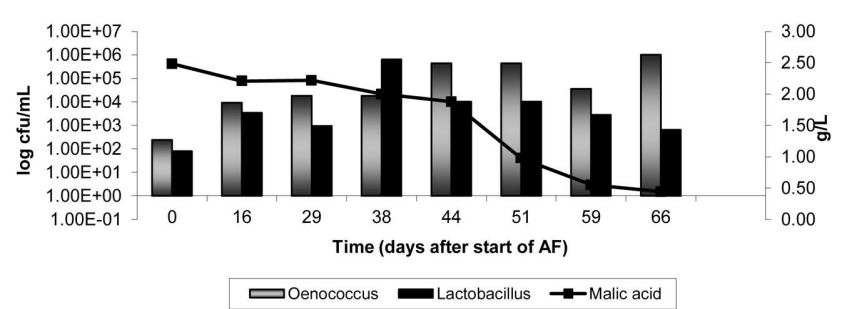

2. Complex yeast nutrient $A$

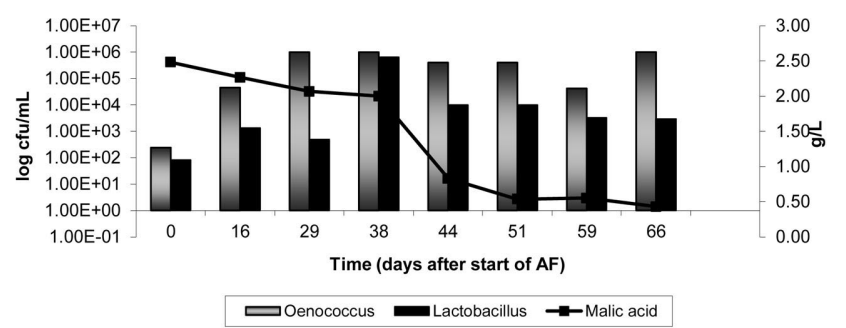

4. Complex yeast nutrient $\mathrm{C}$

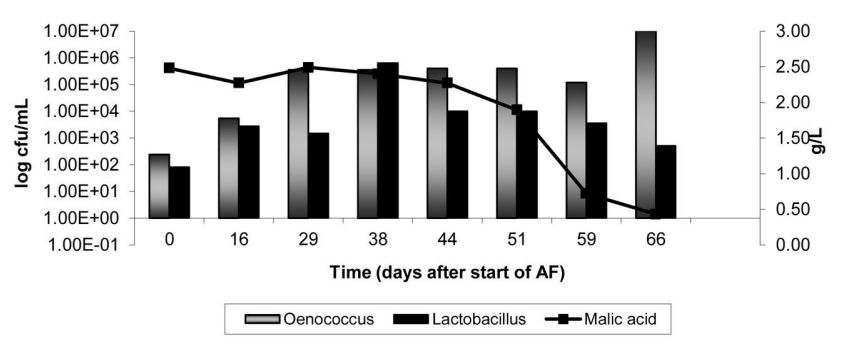

6. Complex MLF nutrient $\mathrm{E}$

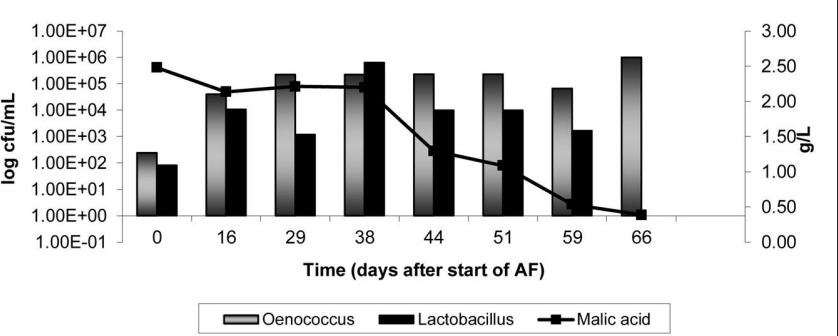

FIGURE 1 | Malolactic fermentation (MLF) (malic acid degradation) and growth of lactic acid bacteria in Cabernet Sauvignon wines. Results are the average of duplicate treatments. AF=alcoholic fermentation.

for all treatments where Lactobacillus species were present could be observed. As with histamine and tyramine, it appears that the Lactobacillus species produced much higher levels of cadaverine when their growth was not preceded by the growth of yeast. When comparing treatment 2 (no complex nutrients in the presence of yeast) and treatments 11 and 12, it is seen that the nutrients could have influenced cadaverine production by LAB when yeast was also present.

\section{INFLUENCE OF MLF INOCULATION TIME ON BIOGENIC AMINES}

In the Pinotage the total concentration of biogenic amines was higher in the co-inoculation treatments than in the sequential treatments (Figure 5). Precisely the opposite was seen in the Shiraz, where the total biogenic amine concentration was higher in the sequential treatments (Figure 6).

All four biogenic amines that were measured could be detected in one or more of the wines. For both inoculation scenarios in the Pinotage and Shiraz, histamine did not increase significantly from before to after MLF in any of the treatments (Figures 5 and 6). No significant production of tyramine occurred in any of the treatments, except for the treatments inoculated with L. hilgardii 1 (Figures 5 and 6). This is not a surprising result. All the strains used in these fermentations were analyzed for the presence of histidine, tyrosine, and ornithine decarboxylases by a multiplex PCR assay (Marcobal et al., 2005) and only this L. hilgardii strain gave a positive result for the tyrosine decarboxylase gene (data not shown). The concentration of tyramine produced by this strain during the sequential inoculation was also higher than what was produced when co-inoculated. In all the other treatments the concentration of tyramine was between 0 and $0.007 \mathrm{mg} / \mathrm{L}$.

Putrescine was the most abundant amine in the analyzed wines. In the Pinotage co-inoculation treatments putrescine increased significantly from before MLF to after AF and MLF (Figure 5). However, in the Shiraz co-inoculation treatments putrescine decreased during the course of these processes (Figure 6). The exact same incidence was seen for cadaverine (Figures 5 and $\mathbf{6}$ ). In the sequential treatments of both cultivars, putrescine concentrations were the same or less than before MLF. For cadaverine, there was also no significant difference in the concentrations before and after MLF in the sequential treatments, except for the uninoculated 


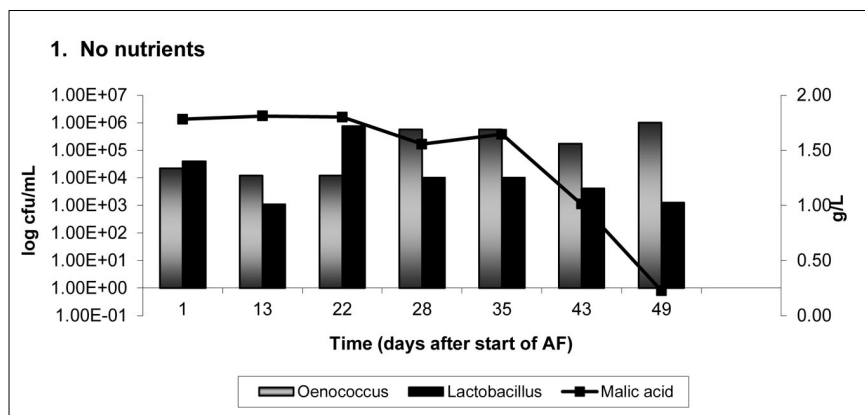

3. Complex yeast nutrient $B$

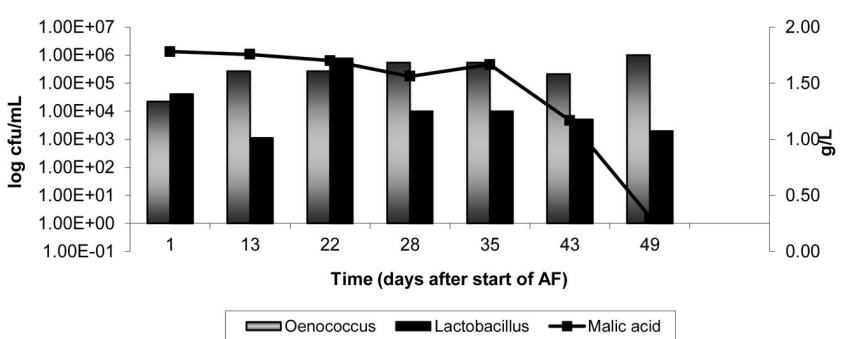

5. Complex MLF nutrient $D$

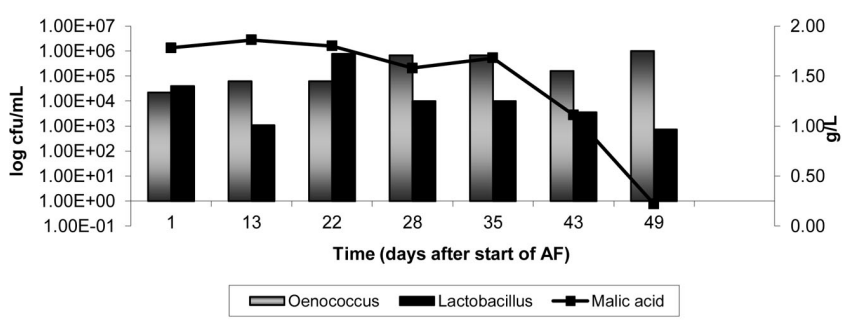

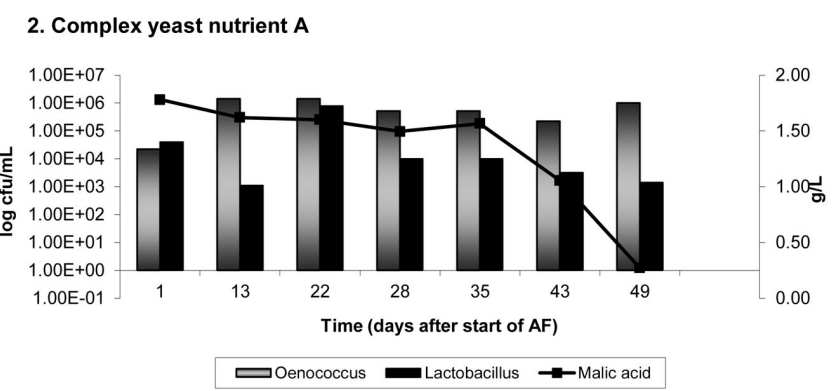

4. Complex yeast nutrient $\mathrm{C}$

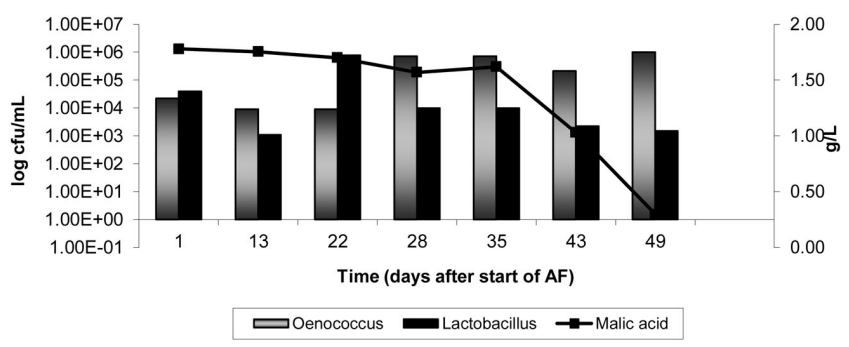

6. Complex MLF nutrient $\mathrm{E}$

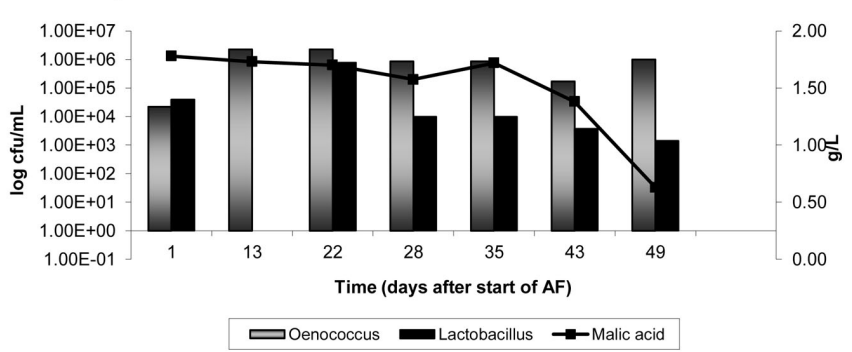

FIGURE 2 | Malolactic fermentation (MLF) (malic acid degradation) and growth of lactic acid bacteria in Shiraz wines. Results are the average of duplicate treatments. AF = alcoholic fermentation.
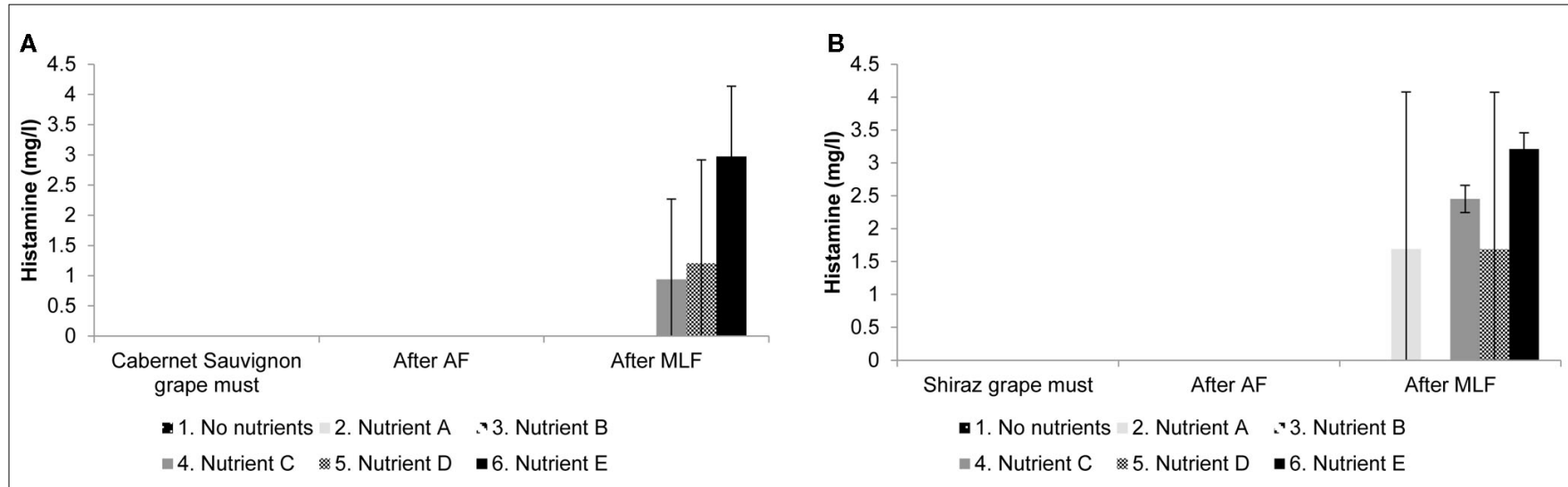

FIGURE 3 | Histamine production in (A) Cabernet Sauvignon and (B) Shiraz wines supplemented with complex yeast or bacterial nutrients. Results are the average of duplicate treatments. 


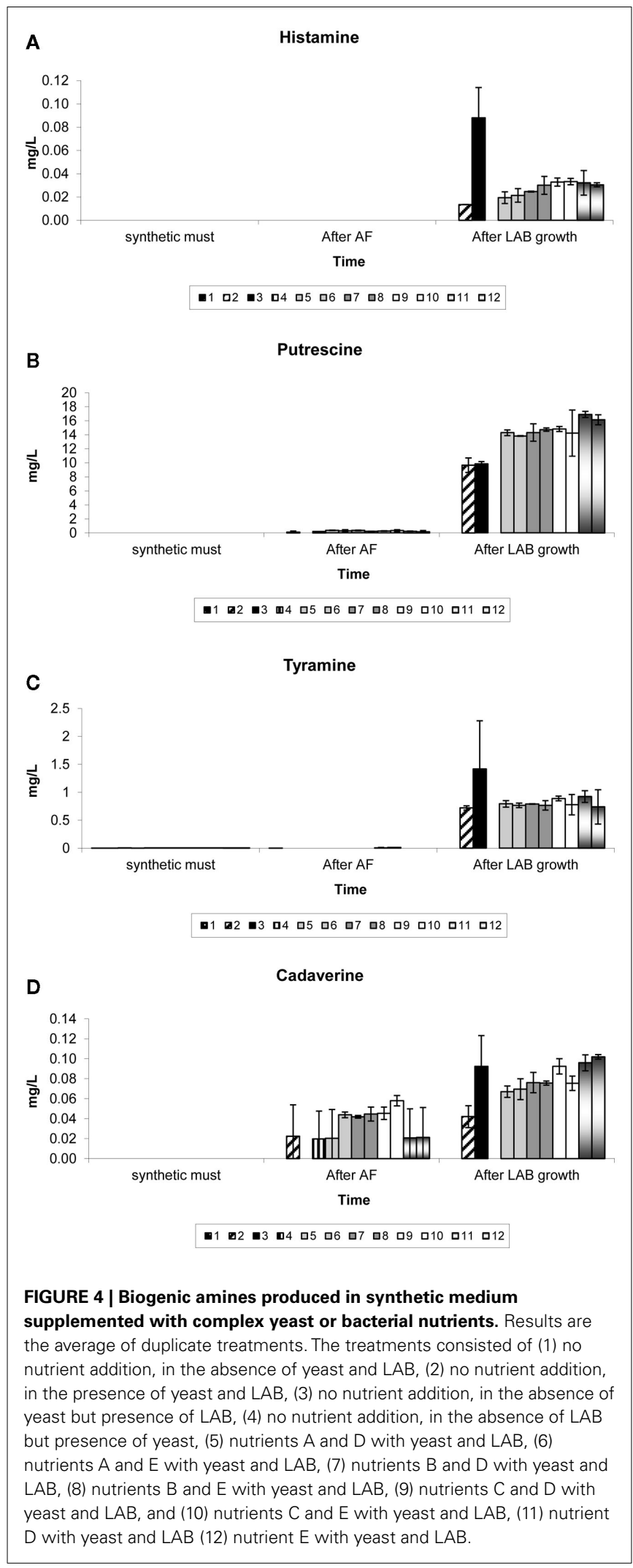

control in the Shiraz that only differed significantly from the concentration before MLF.

\section{DISCUSSION}

\section{GRAPE MUST FERMENTATIONS WITH COMPLEX NUTRIENTS}

Some biogenic amines, particularly polyamines such as putrescine, are often present in grape berries since they are produced by the metabolism of plants (Halász et al., 1994; Bover-Cid et al., 2006). In this study, putrescine was present in the grapes used to assess complex nutrients at relatively high concentrations, at $24.2 \mathrm{mg} / \mathrm{L}$ in Shiraz grape must and $35.7 \mathrm{mg} / \mathrm{L}$ in Cabernet Sauvignon grape must. Vine nutrition, such as potassium deficiencies in the soil, has been linked to elevated concentrations of putrescine (Adams, 1991). In addition, vine nutrition and grape variety are significant contributors to the concentration and composition of grape amino acids, which could determine the final biogenic amine concentration that is found or formed by microorganisms in wine (Soufleros et al., 1998; Herbert et al., 2005).

Histamine was the only biogenic amine showing treatment differences potentially attributable to the presence of complex nutrients. In some treatments we observed large variation in histamine levels between the treatment replicates. Still, it seems possible that there are differences between treatments and that nutrients C, D, and E (Cabernet Sauvignon and Shiraz) and nutrient A (Shiraz only) influenced the final histamine concentration in the wine (Figure 3). Due to the small volumes used in small scale winemaking, it is possible that the inherent heterogeneity in a product such as complex nutrients could have caused the discrepancy in treatment duplicates. Since the increase in histamine occurred during MLF, the formation of this biogenic amine can most likely be attributed to the decarboxylation of histidine by LAB. Of the four biogenic amines analyzed in this study histamine is the most important to human physiology, since it is one of the most biologically active amines (Halász et al., 1994). Histamine can cause hypotension, flushing, and headache (Silla Santos, 1996) as well as abdominal cramps, diarrhea, and vomiting (Taylor, 1986). The levels of histamine produced in some treatment replicates in both cultivars during grape must fermentations supplemented with complex nutrients are above the upper limits suggested for histamine in wine in Germany $(2 \mathrm{mg} / \mathrm{L})$ and Holland $(3 \mathrm{mg} / \mathrm{L}$; Lehtonen, 1996).

During this study, a few trends regarding biogenic amine production in relation to complex yeast and bacterial nutrients could be observed. The production of relatively high levels of histamine in wine during or after MLF (by decarboxylase positive $\mathrm{LAB}$ ) as a result of complex nutrient addition could significantly impact the wholesomeness of the wine and present negative trade implications.

These results obtained in wine are in accordance with a similar study reported in the literature which examined the impact of yeast autolyzate, often a component of complex yeast nutrients, on biogenic amine production during winemaking. The enrichment of Chardonnay must by yeast autolyzate did not lead to an increase of biogenic amines during AF. However, the concentration of biogenic amines, particularly tyramine and cadaverine, were higher after MLF (González-Marco et al., 2006).

Another study that yielded similar results with regards to yeast nutrients was performed by Marques et al. (2008), who tested two commercial fermentation activators. A nutritive factor used for AF was added to the must, and a nutritive factor used for MLF was 


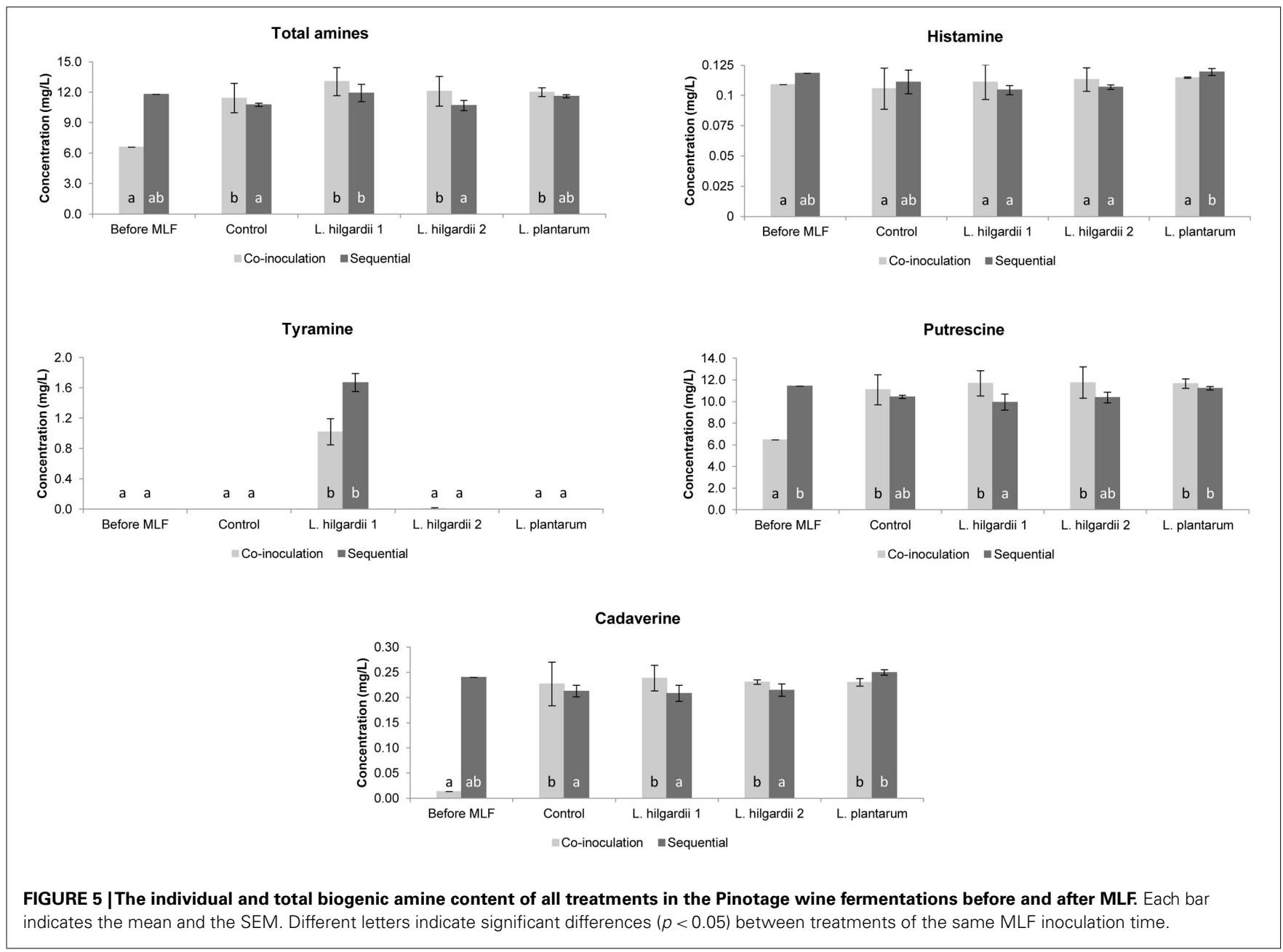

added at the end of AF to the respective treatments. In their study, a slight increase in biogenic amines (particularly isoamylamine and tyramine) resulted from the addition of the AF activator. This increase was especially noticeable at the end of MLF. No significant differences could be observed between wines to which the MLF activator were added and the control wines.

Histamine was produced at higher levels in Shiraz than in Cabernet Sauvignon treatments. This seems to be particularly true for nutrients A and C (Figure 3); however, due to the large variability between replicates this result is probably not significant. Still, it is clear that grape cultivar is a variable that may significantly influence the production of biogenic amines. It is possible that the amino acid composition of the cultivar could influence the amount of available precursors for biogenic amine production, or that the phenolic composition of the grape cultivar could play a role. In another study, using the same grape cultivars but from different vintages and locations, we also found significantly higher histamine production in Shiraz compared to Cabernet Sauvignon (Smit et al., submitted).

\section{SYNTHETIC MEDIUM FERMENTATIONS WITH COMPLEX NUTRIENTS}

From the results presented in Figure 4, it can be concluded that any major contribution to the increase of biogenic amines was contributed by the Lactobacillus species and that they likely produce more histamine, tyramine, putrescine, and cadaverine in an environment that lacks complex nutrients. An explanation for this observation could be that $\mathrm{LAB}$ produce biogenic amines in order to generate metabolic energy or regulate (increase) the $\mathrm{pH}$ of the growth medium (Molenaar et al., 1993). When AF preceded LAB inoculation, biogenic amine production was reduced. It can be proposed that yeast potentially eliminate precursor amino acids from the medium, leading to a reduction of subsequent biogenic amine production. Co-inoculation was not tested in this study.

In synthetic wine medium, it was confirmed that biogenic amines are produced by $\mathrm{LAB}$ and not by yeast. However, the impact complex nutrients appear to have on histamine production in grape must was not confirmed in synthetic medium. In the synthetic grape must experiment, the absence of any complex nutrients seemed to stimulate histamine and tyramine formation by Lactobacillus species (treatment 3). This is contradictory to our result observed in two cultivars of grape must, where histamine production corresponded to the addition of certain nutrients. A relationship seems to exist between yeast and bacterial growth and biogenic amine concentrations produced in the synthetic medium. It appears from this experiment in synthetic medium that the absence of complex nutrients (the medium composition) has a 


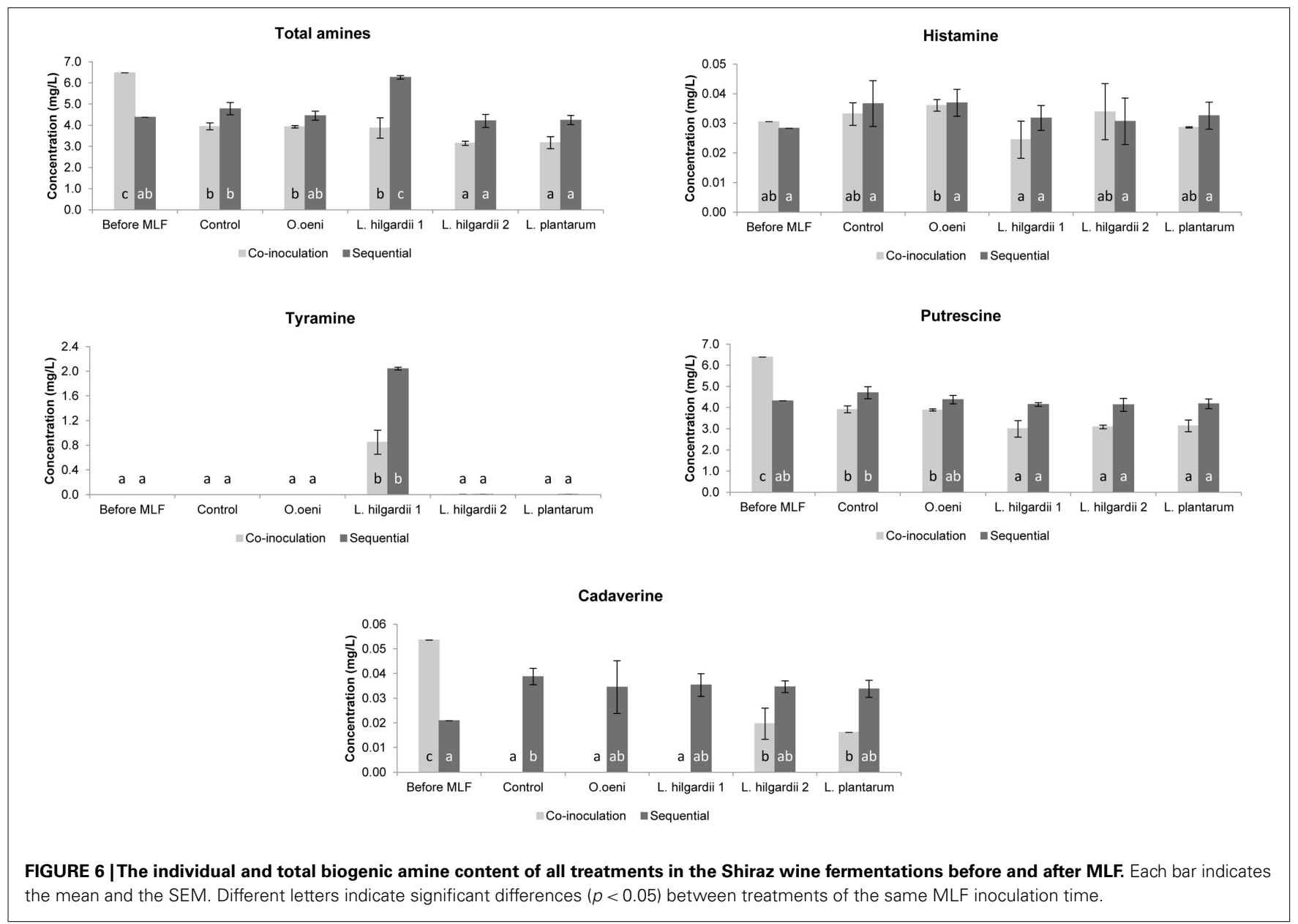

more pronounced impact on biogenic amine production than the presence of any complex fermentation nutrients.

Wine is a complex medium in which many compounds and microorganisms can inhibit the growth and metabolisms of one another or produce synergistic effects that are not observable in a simple chemically defined synthetic medium. It has been observed in a number of studies that biogenic amine production by wine microorganisms in various synthetic media can show a different set of results when evaluated in wine (Guerrini et al., 2002; Granchi et al., 2005; Landete et al., 2007). The synthetic matrix used was perhaps not an ideal model for wine, since it did not promote MLF in the 14-days of the experiment, even though LAB growth occurred.

\section{INFLUENCE OF MLF INOCULATION TIME ON BIOGENIC AMINES}

The evolution of biogenic amines during MLF was monitored to evaluate the impact of different MLF inoculation scenarios on the biogenic amine concentration. Inoculation for MLF simultaneously with AF has been shown to reduce the development of biogenic amines in wine compared to the traditional sequential inoculation (van der Merwe, 2007; Smit and Du Toit, 2011). This seems to be a realistic tool to limit biogenic amine contamination. The inoculated culture may be able to dominate and inhibit the growth of the natural LAB flora and thus the chances of unwanted activities by these bacteria are lessened during the course of AF. Our results support and contradict the findings of van der Merwe (2007) and Smit and Du Toit (2011) where co-inoculation resulted in lower biogenic amine concentrations. The total biogenic amine concentration after MLF in the co-inoculated treatments was lower than before MLF in the Shiraz, but higher in the Pinotage. This contradictory result can possibly be explained by the large number of factors (such as the vintage, geographical region, grape variety, vinification methods, availability of amino acid precursors, etc.) that can influence the formation of biogenic amines in wine (Smit et al., 2008).

Histamine did not increase significantly from before MLF to after MLF in any of the treatments. Thus, the contribution of the malolactic bacteria together with the yeast during co-inoculation and the malolactic bacteria alone in the sequential treatments did not affect the formation of histamine at all.

Significant tyramine formation was only observed in the treatments inoculated with L. hilgardii strain 1 . A PCR assay confirmed that this strain is indeed a tyramine producing strain. It is also important to note that this strain produced more tyramine when it was inoculated sequentially compared to the co-inoculation in both cultivars. Thus, by applying co-inoculation with a LAB strain that is able to produce biogenic amines, could lower the risk of biogenic amine contamination. Moreover, this result highlights 
the importance of inoculating with a starter culture unable to produce biogenic amines.

Of all the amines measured, putrescine was present in the highest concentration. The significant increase in the putrescine and cadaverine concentration in the Pinotage co-inoculated treatments, which was even seen in the uninoculated control treatment, suggests that the yeast had the largest contribution. Exactly the opposite was seen in the Shiraz co-inoculated treatments. A decrease in the amount of these two amines was observed. Several authors reported that yeast can produce biogenic amines during AF (Buteau et al., 1984; Ancín-Azpilicueta et al., 2008; Smit et al., 2008), but the prevailing opinion is that biogenic amines are produced by LAB during MLF (Soufleros et al., 1998; Lonvaud-Funel, 1999). From these results it appears that the inoculated bacteria did not affect the biogenic amine content of the wine during MLF. In the sequential inoculated treatments, where the involvement of the LAB in biogenic amine formation alone can be seen, all amines were present in the same or significantly lower concentration as before inoculation for MLF, except for cadaverine in the uninoculated control treatment in the Shiraz and tyramine in both cultivars. Thus, the relationship between biogenic amines and MLF is debatable in this dataset. In some previous studies biogenic amines increased during MLF (Marcobal et al., 2006; Izquierdo Cañas et al., 2008), while it decreased in others (Buteau et al., 1984). This inconsistency in results can be ascribed to the wide variety of factors that can influence biogenic amine production in wine, especially the fact that different species of yeast and LAB as well as strains of the same species differ in their ability to produce biogenic amines (Ancín-Azpilicueta et al., 2008; Marques et al., 2008; Smit et al., 2008).

In conclusion, when comparing the observations made in real grape must and synthetic grape must supplemented with complex nutrients, it is clear that two different results were obtained.

\section{REFERENCES}

Adams, D. O. (1991). "Accumulation of putrescine in grapevine leaves showing symptoms of potassium deficiency or spring fever," in Proceedings of the International Symposium on Nitrogen in Grapes and Wine, ed. J. Rantz (Davis, CA: American Society for Enology and Viticulture), 126-131.

Alberto, M. R., Arena, M. E., and Manca de Nadra, M. C. (2002). A comparative survey of two analytical methods for identification and quantification of biogenic amines. Food Control 13, 125-129.

Ancín-Azpilicueta, C., González-Marco, A., and Jiménez-Moreno, N. (2008). Current knowledge about the presence of amines in wine. Crit. Rev. Food Sci. Nutr. 48, 257-275.

Bach, B., Colas, S., Massini, L., Barnavon, L., and Vuchot, P. (2011). Effect of nitrogen addition during alcoholic fermentation on the final content of biogenic amines in wine. Ann. Microbiol. 61, 185-190.
Bely, M., Sablayroles, J., and Barre, P. (1990). Description of alcoholic fermentation kinetics: its variability and significance. Am. J. Enol. Vitic. 40, 319-324.

Bover-Cid, S., Iquierdo-Pulido, M., Mariné-Font, A., and Vidal-Carou, M. C. (2006). Biogenic mono-, diand polyamine contents in Spanish wines and influence of a limited irrigation. Food Chem. 96, 43-47.

Buteau, C., Duitschaver, C. L., and Ashton, G. C. (1984). A study of biogenesis of amines in a Villard noir wine. Am. J. Enol. Vitic. 35, 228-236.

Downing, L. (2003). Characterisation of Biogenic Amine-Encoding Genes in Lactic Acid Bacteria Isolated from South African Wine. M.Sc. thesis, Stellenbosch University, Private Bag X1, 7602 Matieland, Stellenbosch.

González-Marco, A., Jiménez-Moreno, N., and Ancín-Azpilicueta, C. (2006). Influence of addition of yeast autolysate on the formation of amines in wine. J. Sci. Food Agric. 86, 2221-2227.

From the real grape must experiment we could conclude that complex nutrient additions have the potential to increase histamine. A similar result was observed for amines putrescine and cadaverine in synthetic grape must. However, from the synthetic grape must experiment, it appears that biogenic amine production by LAB is influenced largely by the combination or succession of microorganisms and not always stimulated by the presence of complex nutrients, as noted for histamine and tyramine. Importantly, it is clearly observed that the presence of decarboxylase positive $\mathrm{LAB}$ is associated with biogenic amine increase in both the synthetic and real grape must experiments. It is therefore recommended to inoculate for MLF with commercial starter cultures that do not contain harmful decarboxylase activities. From our results in real grape must it appears that complex nutrients could influence wine wholesomeness negatively by stimulating biogenic amine production. However, from our results in synthetic must it seems that a lack of nutrients could be equally or more harmful in this regard. Thus, the judicious use of complex yeast and bacterial nutrients, used in combination with a decarboxylase negative $\mathrm{LAB}$ strain is recommended.

By inoculating for MLF simultaneously with AF has shown to reduce the incidence of biogenic amines in previous studies. Our results agree and disagree with these findings. However, by applying co-inoculation with a strain (L. hilgardii 1 ) that is capable of producing tyramine resulted in a lower concentration of the specific amine compared to when it was sequentially inoculated. These results also emphasize the importance of using decarboxylase negative $\mathrm{LAB}$ strains for MLF.

\section{ACKNOWLEDGMENTS}

The authors thank the National Research Foundation, Winetech, Kron Morelli srl, and THRIP for financial support.

Granchi, L., Romano, P., Mangani, S., Guerrini, S., and Vincenzini, M. (2005). Production of biogenic amines by wine microorganisms. Le Bulletin de l'OIV 78, 595-609.

Guerrini, S., Mangani, S., Granchi, L., and Vincenzini, M. (2002). Biogenic amine production by Oenococcus oeni. Curr. Microbiol. 44, 374-378.

Halász, A., Baráth, Á., Simon-Sarkadi, L., and Holzapfel, W. (1994). Biogenic amines and their production by microorganisms in food. Trends Food Sci. Technol. 5, 42-49.

Herbert, P., Cabrita, M. J., Ratola, N., Laureano, O., and Alves, A. (2005). Free amino acids and biogenic amines in wines and musts from the Alentejo region. Evolution of amines during alcoholic fermentation and relationship with variety, sub-region and vintage. J. Food Eng. 66, 315-322.

Hernández-Orte, P., Bely, M., Cacho, J., and Ferreira, V. (2006). Impact of ammonium additions on volatile acidity, ethanol, and aromatic compound production by different Saccharomyces cerevisiae strains during fermentation in controlled synthetic media. Aust. J. Grape Wine Res. 12, 150-160.

Izquierdo Cañas, P. M., García Romero, E., Gómez Alonso, S., Fernández González, M., and Palop Herreros, M. L. L. (2008). Amino acids and biogenic amines during spontaneous malolactic fermentation in Tempranillo red wines. J. Food Compost. Anal. 21, 731-735.

Landete, J. M., Ferrer, S., and Pardo, I. (2007). Biogenic amine production by lactic acid bacteria, acetic bacteria and yeast isolated from wine. Food Control 18, 1569-1574.

Lehtonen, P. (1996). Determination of amines and amino acids in wine a review. Am. J. Enol. Vitic. 47, 127-133.

Leitão, M. C., Teixeira, H. C., Barreto Crespo, M. T., and San Romão, M. V. (2000). Biogenic amines occurrence 
in wine: amino acid decarboxylase and proteolytic activities expression by Oenococcus oeni. J. Agric. Food Chem. 48, 2780-2784.

Lerm, E., Engelbrecht, L., and Du Toit, M. (2010). Malolactic fermentation: the ABC's of MLF. S. Afr. J. Enol. Vitic. 31, 186-212.

Lonvaud-Funel, A. (1999). Lactic acid bacteria in the quality improvement and depreciation of wine. Antonie Van Leeuwenhoek 67, 317-331.

Marcobal, Á., De las Rivas, B., MorenoArribas, V., and Muñoz, R. (2005). Multiplex PCR method for the simultaneous detection of histamine-, tyramine- and putrescine-producing lactic acid bacteria in foods. J. Food Prot. 68, 874-878.

Marcobal, Á., Martínez-Álvarez, P. J., Polo, M. C., Muñoz, R., and MorenoArribas, V. (2006). Formation of biogenic amines throughout the industrial manufacture of red wine. J. Food Prot. 69, 397-404.

Marques, A. P., Leitão, M. C., and San Romão, M. V. (2008). Biogenic amines in wines: influence of oenological factors. Food Chem. 107, 853-860.
Molenaar, D., Bosscher, J. S., ten Brink, B., Driessen, A. J. M., and Konings, W. N. (1993). Generation of a proton motive force by histidine decarboxylation and electrogenic histidine/histamine antiport in Lactobacillus buchneri. J. Bacteriol. 175, 2864-2870.

Moreno-Arribas, M. V., Smit, A. Y., and du Toit, M. (2010). "Biogenic amines and the winemaking process," in Understanding and Managing Wine Quality and Safety, ed. A. G. Reynolds (Cambridge: Woodhead Publishing Limited), 494-522.

Ribérau-Gayon, P., Dubourdieu, D., Donèche, B., and Lonvaud, A. (2006). "The microbiology of wine and vinifications," in Handbook of Enology, 2nd Edn, Vol. 1, ed. P. Ribéreau-Gayon (Chichester: Wiley), 85-89.

Silla Santos, M. H. (1996). Biogenic amines: their importance in foods. Int. J. Food Microbiol. 29, 213-231.

Smit, A. Y., and Du Toit, M. (2011). Evaluating the influence of malolactic fermentation inoculation practices and ageing on lees on biogenic amine production in wine. Food Bioprocess Technol. doi:10.1007/s11947-011-0702-8
Smit, A. Y., Du Toit, W. J., and Du Toit, M. (2008). Biogenic amines in wine: understanding the headache. S. Afr. J. Enol. Vitic. 29, 109-127.

Soufleros, E., Barrios, M., and Bertrand, A. (1998). Correlation between the content of biogenic amines and other wine compounds. Am. J. Enol. Vitic. 49, 266-278.

Taylor, S. L. (1986). Histamine food poisoning, toxicology and clinical aspects. Crit. Rev. Toxicol. 17, 91-128.

van der Merwe, H. (2007). The Evaluation of Malolactic Fermentation Starter Cultures Under South African Winemaking Conditions. M.Sc. thesis, Stellenbosch University, Private Bag X1, 7602 Matieland, Stellenbosch.

Vidal-Carou, M. C., AmbatlleEspunyes, A., Ulla-Ulla, M. C. and Mariné-Font, A. (1990a). Histamine and tyramine in Spanish wines: their formation during the winemaking process. Am. J. Enol. Vitic. 41, 160-167.

Vidal-Carou, M. C., Codony-Salcedo, R., and Mariné-Font, A. (1990b). Histamine and tyramine in Spanish wines: relationships with total sulfur dioxide level, volatile acidity and malolactic fermentation intensity. Food Chem. 35, 217-227.

Conflict of Interest Statement: The authors declare that the research was conducted in the absence of any commercial or financial relationships that could be construed as a potential conflict of interest.

Received: 21 December 2011; paper pending published: 05 January 2012; accepted: 14 February 2012; published online: 13 March 2012.

Citation: Smit AY, Engelbrecht $L$ and $d u$ Toit M (2012) Managing your wine fermentation to reduce the risk of biogenic amine formation. Front. Microbio. 3:76. doi: 10.3389/fmicb.2012.00076

This article was submitted to Frontiers in Food Microbiology, a specialty of Frontiers in Microbiology.

Copyright (C) 2012 Smit, Engelbrecht and $d u$ Toit. This is an open-access article distributed under the terms of the Creative Commons Attribution Non Commercial License, which permits noncommercial use, distribution, and reproduction in other forums, provided the original authors and source are credited. 